March - 2007

\title{
Online Distance Teaching of Undergraduate Finance: A Case for Musashi University and Konan University, Japan
}

\author{
Keiichi Kubota \\ Faculty of Economics, Musashi University \\ Tokyo, Japan \\ Kiyoshi Fujikawa \\ Faculty of Economics, Konan University, \\ Kobe, Japan
}

\begin{abstract}
We implemented a synchronous distance course entitled: Introductory Finance designed for undergraduate students. This course was held between two Japanese universities. Stable Internet connections allowing minimum delay and minimum interruptions of the audio-video streaming signals were used. Students were equipped with their own PCs with pre-loaded learning materials and Microsoft Excel exercises. These accompanying course and exercise materials helped students comprehend the mathematical equations and statistical numerical exercises that are indispensable to learning Introductory Finance effectively. The general tendency for students, not to raise questions during the class hours in Japan, however, was found to be a big obstacle. As such, motivational devices are needed and should ideally be combined to promote interaction between the e-classrooms.
\end{abstract}

Keywords: Distance learning; online teaching; wide range LAN; e-Japan; Microsoft Excel exercises

\section{Introduction}

This paper describes the authors' experiences in designing and teaching an online, synchronous distance education undergraduate course, Introductory Finance. Two universities, Musashi University in Tokyo and Konan University in Kobe, located 400 miles apart, were involved in this project and the foci of this descriptive case study. Prior to embarking on this joint-project, both universities signed formal agreements to share resources for the development of this undergraduate finance course. Course developers involved in this project also visited the other campus frequently to familiarize themselves with both universities' institutional and student cultures, systems, and protocols. As such, all involved in developing this synchronous e-course were fully aware of each other's unique differences in terms of curriculum, student needs, and institutional cultures at both universities BEFORE the project was built. We believe this 'period 
of acquaintance' helped enormously in developing this new online, synchronous distance education course.

\section{How this paper is arranged}

First, we overview the current e-Learning environment in Japan and define the mission of our project within the context of this environment. We then report our experience in developing and teaching Introductory Finance at two privately funded Japanese universities using an online, synchronous teaching environment comprised of streaming video relayed between the 'main classroom' at Musashi University in Tokyo and the 'remote classroom' at Konan University in Kobe. Specifically, we focus on the system and instructional design used, and describe our preparations for teaching online and project implementation. Described are the physical classroom environments and instructional devices employed, followed by a descriptive assessment of the project including problems encountered. We then suggest some possibilities for future improvement, from which students and academics may draw conclusions for further research.

\section{e-Learning Environments in Japan and Our Project's Mission}

Although Japan is one of the most advance users and developers of IT hardware and technology in the world, and such technologies are employed in all sectors of the Japanese economy, including the academic sector, Japan's use of the Web-based technologies lags behind that of many other nations. For example, according to one recent report, Japan ranked 24th in the world and seventh in the Asia-Pacific region in its use of the Internet (The Economist Intelligent Unit \& IBM, 2003). It can thus be inferred from this report that e-Learning is not well developed in Japan. Moreover, some suggest that Japanese people, in general, are culturally averse to distancelearning (Yoshida, 2004). We will discuss these later points within the context of our experiences in more detail later in this paper.

To redress problems of Web-based IT diffusion in both its industrial and educational sectors, in January 2001 the Japanese government established the 'Strategic Headquarters for the Promotion of an Advanced Information and Telecommunications Network Society' - a.k.a., IT Strategic Headquarters - and a project called "e-Japan”. Included in the IT Strategic Headquarters' and eJapan's formally stated objectives are the development of Web-based IT education, e-Learning, and the development of human resources necessary to support and sustain such endeavors. Their target date for introducing Web-based IT education was 2005 (IT Strategic Headquarters, 2001).

Prior to implementing its e-Japan project, the Japanese Ministry of Education, Culture, Sports, Science and Technology (MEXT) had approved Master's programs using distance education in 1998 (Wong \& Yoshida, 2001). Three years later, blended undergraduate programs were made available using a 'blend' of both distance and traditional modes, allowing undergraduates to earn up to 60 'distance' credits towards their minimum 124 credit Bachelor's degree (Jung \& Suzuki, 2006).

In light of the general trends in e-Learning in Japan described above, we rationalized and developed our project along these lines.

- We followed the initiatives as set forth by the Japanese Cabinet in its “e-Japan” project. 
- We strategically aligned the projects' objectives with those of the Japanese University Association for Computer Education (JUCE) and MEXT, two organizations that have been proactively promoting Japan's Web-based IT strategy at the university level for several years.

- We followed the natural progression of this trend. Indeed, because both universities were members of the "five-university league” (Gakushuin University, Seikei University, Seijo University, Konan University, and Musashi University), developing a joint distance learning project between two of these universities (Musashi and Konan) can be viewed as a logical step.

In defining the mission of our e-Learning project, we sought to address the mandates of e-Japan, JUCE, and MEXT. In spite of this, it must be made clear that we embarked upon this e-Learning project facing considerable cultural constraints, specifically biases against distance education in general. Indeed, some have argued that Japanese people value face-to-face education over that of distance education, and thus learning distance as a whole is viewed as inferior (Yoshida, 2004; Jung \& Suzuki, 2006). Indeed, either by personal choice or simply by adhering to long established cultural norms, Japanese students typically avoid asking questions during lectures for fear of drawing attention to themselves. In Japanese culture - notably in classroom settings where teachers are venerated and expected to take command - students prefer to look and act 'anonymously.'

Wong and Yoshida (2001) report on previous implementations of e-Learning and distance learning at two Japanese universities. Wong and Yoshida's research, however, focuses on parttime students. Some argue that part-time working adult students are a unique subset of the larger student population, a sub-set that is highly motivated to learn as compared to younger, full-time students (Kawachi, 2005a). Accordingly, we submit that any findings drawn from our case study cannot be generalized to all Japanese undergraduate students.

Jung and Suzuki (2006) discuss how conventional face-to-face at a Japanese Liberal Arts College was 'blended' with online learning components to arrive at better learning outcomes, including enhanced classroom interactions, efficient classroom management, and effective information sharing between and among students and instructors. As of 2007, the International Christian University uses Blackboard, WebCT and Moodle to promote blended learning in its undergraduate and graduate courses (ICU, n.d.). It is conjectured, however, that because The International Christian University is a bilingual university (Japanese and English) its students and instructors are bilingual, and thus much more likely to have been exposed to e-Learning through its use of Western-made e-Learning management system and e-teaching materials. As such, it is suggested that while these reports are very valuable and provide great insights, the International Christian University's experiences in e-Learning are likely not typical of e-Learning in Japanese universities in general, wherein most topics are taught exclusively in Japanese to unilingual Japanese students who have had limited or no exposure to e-Learning. As such, it is suggested our experiences as described in this paper will add to the broader body of literature examining Japanese-only undergraduate e-Learning and distance learning.

\section{Scope and Subjects}

The focus of this study is an undergraduate course distributed between two conventional private undergraduate universities in Japan, located 400 miles apart. 
The subjects of this case study were full-time native Japanese university-level undergraduate students. The students' are lectured in their mother tongue - Japanese - and the teaching and learning materials used are written and administered in Japanese. While some Japanese universities have engaged in online distance learning initiatives before, such activities were developed within the context of one university and typically delivered between that university's main campus and its' satellite campuses (e.g., Chuo University and Keio University). The case study reported here, however, is unique in that two completely different universities collaborated in the design and delivery of one online synchronous Introductory Finance course to undergraduate students.

Both classrooms - the main and remote classrooms - were equipped with personal computers (PCs). The computer to student ratio was 1:1, allowing students increased command over their online learning environment. The PCs were configured to allow students to participate in the synchronous, online classes with the other classmates located at the other university.

The authors' academic backgrounds are in economics and finance - not education or pedagogy. Although the authors are not instructional designers, both were involved in the design of the program. Both taught the online synchronous program. Similarly, the teaching assistants used in this project also had backgrounds in economics and finance.

\section{Project Analysis, Design, and Development}

Both universities began discussing this distance-education project in May 2001. As mentioned previously, the project was guided by the objectives outlined in e-Japan and were fully supported by both universities. Moreover, as academics with finance and economic as our scholarly background, we were unfamiliar with course design strategies. As such, from the beginning we attempted to inform our project with established instructional design principles and e-Learning assessments (Shimizu, 2005). Shimizu (2005), for instance, suggests that instructional design for e-Learning and distance learning use: 1) analysis, 2) design, 3) development, 4) implementation, and 5) evaluation. Kawachi (2005a) also emphasizes the importance of using multimedia for learning. Koontz, Li and Compora (2006) further cite the need to follow a concrete model for realizing effective distance education outcomes; they also voice concern that "programs and course are being implemented without following an established system design process" (Koontz, et al., 2006, p. 39). Our synchronous, online distance teaching pilot project, therefore, attempted to address the problems of e-Learning as described by Shimizu (2005), Kawachi (2005a) and Koontz and colleagues (2006). As such, we developed the Introductory Finance online eLearning course in close cooperation with faculty and computing staff at both universities. We similarly allowed adequate time to deal with anticipated and unanticipated problems.

We designed our online synchronous distance-teaching environment with a 1:1 student-tocomputer ratio. Each PC was configured so students could participate in their classroom three ways: 1) a live video feed from the main classroom at Musashi University; 2) a view of the instructors' computer screen located in the central classroom; and 3) a live video feed from the remote classroom at Konan University. The combinations of video streams - from large to small streaming video shots - depended on where an individual student's classroom was located (i.e., main classroom or remote). Online Microsoft Excel exercises were also administered synchronously online, a factor we opine enhanced students' comprehension of the learning objectives of the course. 
Because students' learning styles vary considerably, students' learning process and their comprehension level must be evaluated in a flexible manner. As such, we structured student assessments following eight core elements (Koontz, et al., 2006): 1) environment, 2) emotional, 3) structure, 4) sociological, 5) physical, 6) instructional, 7) response, and 8) cognitive strategies used.

To predict our project's outcomes prior to implementation, we decided to refine our project's predicted outcomes by breaking our learning situation into two high-level dimensions: 'favorable' versus 'unfavorable' (Shimizu, 2005).

To predict our projects 'favorable' dimensions, we needed to determine our course's inherent strengths. We determined that the 1:1 student-to-PC ratio and students' online real-time, hands-on use of Microsoft Excel would greatly assist in their comprehension of the subject matter being taught, and thus would be on the 'favorable' side of the two dimensions. In terms of Koontz's eight elements, we determined these favorable dimensions aligned to Kootz's element 1 (environment), element 3 (structure), element 5 (physical), and element 6 (instructional).

To predict our projects 'unfavorable' dimensions, we needed to determine our course's inherent weaknesses. For the 'unfavorable' dimensions, we knew in advance that the students were from two different universities and would come to the course prepared with different sets of course prerequisites. Moreover, the students would be historically mired a culture wherein they would be more apt to defer to their instructor and seek to remain 'anonymous' and not draw attention to themselves. These 'unfavorable' dimensions align with Koontz's element 1 (environment) and more pointedly to element 4 (sociological).

Accordingly, element 4 (sociological) was deemed from the outset to be our greatest inherent weakness. Because these students would be new to distance learning environments, they would more likely be subjected to experience intense feelings of distress and unease, which in turn links downward to Koontz's element 2 (emotional) and 7 (response). Put simply, we knew in advance that the students enrolled in this project would need lot of courage to ask questions and a lot of encouragement to get them to talk and participate. In other words, we were asking students to remove themselves from their timeworn 'emotional' safety net of anonymity and place themselves squarely in center stage.

\section{Project Case Study}

\section{Project preparation, system configurations, and implementation}

In 2000, the Deans of the Faculty of Economics at Musashi University and Konan University met in Kobe Japan to discuss the joint e-Learning project in detail. At that time, MEXT had started to accredit distance learning environments. To receive accreditation, however, certain conditions and criteria had to be met. One criterion, for instance, was that before distance programs could be officially accredited by MEXT, they had to be staffed with qualified teaching personnel. Another criterion was that the distance e-Learning environment also had to be robust enough to support synchronous, real-time interaction between students and their teachers. Put simply, asynchronous distance course delivery would not meet the criteria needed to receive official accreditation. This was a significant technological constraint. 
Indeed, faced with the demands of this criterion, it was determined by the computing staff at both universities that the Internet infrastructure in Japan was not yet very robust. Faced with this technological constraint, it was determined that our first priority was to maintain stable Internet connections. As such, we chose to use a $10 \mathrm{Mbps}$ wide area LAN to connect directly between the two universities. We also chose the same Polycom 4000 cameras to minimize the stress of coordinating between these two equipments (See Figure 1).

Figure 1. System Chart of Musashi University's 8501 classroom

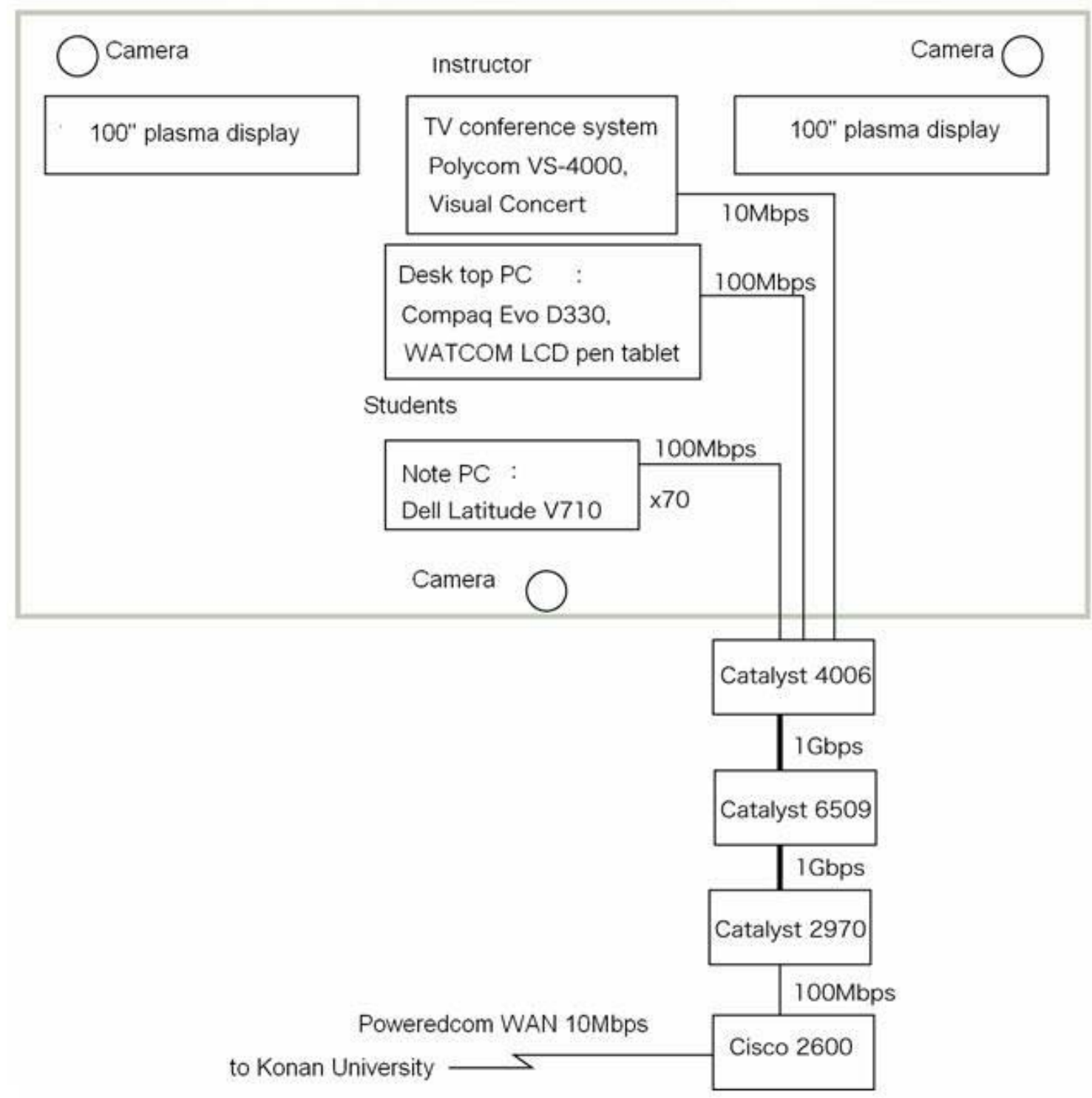

Because both universities were well aware of each other's educational needs and constraints, it was decided that the 'shared course' could not be too difficult. Musashi University, which offers a wide range of Finance courses, suggested that a course in Introductory Finance would be best. Konan University, on the other hand, felt that an Asian (Chinese) economy course would be more appropriate (Konan University is renown for International Economics). Ultimately, after much 
discussion and testing, Introductory Finance was chosen as a course to be taught from Musashi University.

2001 was spent testing streaming signals using hypothetical e-Learning environments. The three vendors, Sony, Panasonic, and Hitachi, provided much needed equipment to conduct these experiments. We were extremely thankful to have such cooperation from these private-sector firms, because the cost of this equipment to support e-Learning was extremely expensive at the time. When the various hypothetical experiments were found to be successful, both universities agreed to exchange online courses and the presidents of the two universities signed a formal agreement to exchange classes using synchronous e-Learning delivered at a distance.

The faculty and staff involved were very excited and wanted to get started straight away; however, as a strictly precautionary measure, we continued with our hypothetical experiments for one more term. Such precaution proved to be prudent, as we soon discovered the streaming audio signals tended to become distorted and, at times, the streaming audio-visual signal dropped altogether. The vendors and the computing staff from both universities worked collaboratively to determine and address the root cause of various technological problems as they became apparent. For instance, at Konan University some of the routers needed to be updated to allow the sending of streaming audio-video signals. Indeed, we discovered that whenever these signals were dropped and lost, the digital packets of information were also lost. At Musashi University, it was determined that the routing of the LAN needed to be changed as well. Once these technological problems were resolved, we were then ready to start the actual design of the course, Introductory Finance. By autumn 2002, our distance e-Learning teaching was officially accredited.

\section{Classroom environment and instructional devices used}

We used a dedicated wide area LAN supported by the 'Internet Initiative Japan' to connect the eLearning classroom at Musashi University to the e-Learning classroom at Konan University. The classrooms at both locations were equipped with PCs resulting in a 1:1 computer-to-student ratio. One large sized projection screen was located the at front each classroom and configured to stream three videos of classroom interaction: two images were broadcast via Polycom cameras situated at both universities e-Learning suites, and an image of the instructor's PC screen (see Figure 1). Three controllable Polycom 4000 cameras were strategically situated in the Musashi classroom and two Polycom 4000 (one stationary and one portable) cameras were situated at Konan University. Teaching assistants at both universities were trained to operate the Polycom 4000 cameras and taught to anticipate students' participation in the e-classrooms. For instance, during the final exam, the teaching assistant at Konan University panned the camera across that university's e-classroom to allow the instructor at Musashi University to monitor the exam.

The lecture component of this course, delivered and facilitated by two economics professors, was broadcast from Musashi University. Konan University provided the professors.

Because 'first time' students were more likely to be hesitant to ask questions due to cultural norms requiring them to remain 'anonymous,' coupled with the pressure of learning how to learn in an eLearning environment, full professors were used to teach the class. Indeed, it was felt that the students were more likely to respect and respond to professors' words of encouragement over that of teaching personnel with lower academic qualifications.

Numerical examples and exercises are indispensable. As such, all students were equipped with their own PC. The first instructor taught the concept of the present value and optimal portfolio 
formation based on 'beta' coefficients (Kubota, 2001). Microsoft Excel was used to teach 'present values,' while 'optimal portfolio formation' was taught using Excel's 'Solver' and 'OLS regression' tools. Students were also taught 'futures and option pricing theory' (Black \& Scholes, 1973) using the numerical generator for normal distributed variables in Excel. As such, students used Microsoft Excel to meet the learning outcomes of Introductory Finance.

The e-classrooms met once a week for 90 minutes, which is typical of most higher education courses in Japan. Students told us that watching the continuous 90 minute lecture broadcast resulted in eyestrain and boredom, more so for the 'receiving' party at the remote classroom at Konan University. To remediate this problem, we sought to combine both the lecture session and the Excel exercise session within the 90 minute sessions. We found that this tactic of combining the lecture with the hands-on use of Excel encouraged students to raise questions, particularly from the remote classroom at Konan University. Still, we regretfully must report that students enrolled in this e-Learning course did not ask questions as much as we had hoped. To address this problem, we found that questions must be raised straight away at the beginning of the course, questions that encourage interaction between the two classrooms, with particular focus paid more on the remote classroom to encourage their participation. Such interaction was also discovered to be very necessary, as it alerted instructors to detect and correct technological issues such as distortions in volume.

It must be stressed, however, that technology did not form the basis of interaction between instructors at the two different universities. To get the classrooms flowing, sometimes the two instructors engaged in heated debate and encouraged students to get involved in the discussion, if only to motivate the students in both e-classrooms. Now that we have been teaching this course for three years, and have reached a level of comfort, it was decided that instructors were not needed at the remote e-classroom. Indeed, the instructor in the host classroom had acquired enough expertise to keep both classrooms active.

\section{Interpretations and evaluation}

Musashi University has been continuously administering and collecting teacher evaluation questionnaires from students enrolled in the Introduction to Finance e-class. While we are not at liberty to disclose the 'actual' content of these surveys, the broad data collected indicate that students enrolled in this e-course were generally satisfied, findings that are comparable to or better than other traditional courses in terms of use of course syllabus, use of projectors, voice clarity, and the use of learning aiding materials such as the PC exercises. Moreover, because a sizable portion of course e-contents prepared learning objects such as PowerPoint presentations, Mathematical files, Excel exercises, these learning objects were also highly assessed by the students.

Students also reported having experienced significant psychological stress. However, we feel that this finding is likely deceptive. Indeed, the e-classroom students could be more stressed by mathematical exercises than the e-Learning aspects of their course. Still, when the students were questioned if they would recommend this e-course to a friend, many reported that they would not recommend taking it, a score that is below that reported in similar courses taught in traditional classroom settings. So, while it is difficult to ascertain whether this difficulty arose in 'course content' or 'distance e-Learning' per se, we still believe the 'course contents' were the more likely explanation for students reluctance to recommend this e-course. We say this because economic students in Japan are typically not well equipped to handle the mathematical or statistical exercises they need to do well on this course, as they are not exposed to these concepts until they 
reach post secondary education. So, while further research is clearly needed (i.e., whether students exposure to statistics is best taught synchronously online versus in traditional face-toface settings), we strongly suspect latter is the case - because most students are grappling with these mathematical concepts for the very first time, they are more apt to experience and therefore report 'stress.'

Nonetheless, previous research by Jung and Suzuki (2006) shows that students tend to discourage others from taking synchronous online courses and instead placing emphasis on traditional courses offered face-to-face as the more preferable option. Indeed, sociological traits specifically element 4 in Koontz's eight core elements (Koontz et. al., 2006) - students are not to ask questions and instead show respect to instructors, which is typical for many Asian countries. In Japan, in particular, most students are reluctant to ask questions and tend to gather and ask questions among each other after the class ends (Koontz, 2006).

The technology used to support the e-classroom could also inadvertently chill interaction and students' learning experience. Use of multiple cameras distributed between two classrooms and the use of microphones and PC-based technologies could very well intimidate many students. This fact, therefore, can be classified as deterrence to learning - element 7, response, according to the definition offered by Koontz and colleagues (2006).

Japanese students' reluctance to ask questions, while not surprising, still made our distance learning endeavor very difficult, indeed. We opine that this aspect alone is likely the most difficult aspect teaching courses online synchronously. In particular, we feel that undergraduate students have yet to learn how to question the validity of 'facts,' theories or opinions, and are thus more apt to benefit less from synchronous e-Learning classes taught at the undergraduate level. In sum, we have serious reservations about success of undergraduate level online synchronous courses in general, an opinion that is supported by others. For instance, Jung and Suzuki (2006) have suggested that blended learning is one viable solution that can improve this dilemma. We further opine that this may, in fact, be the most significant reason why distance online learning is not yet well received in Japanese society - over that of costs involved in building the infrastructure needed to support synchronous e-Learning as envisioned by the Japanese government.

In spite of these aforementioned concerns, we feel that sound instructional design principles must be used (Shimizu, 2005). For example, through trial and error, we found that if students were asked to present their solutions on various exercises to their fellow students at the other location, this alone greatly enhanced discussion and interaction between the two groups. Such interaction must be designed purposely and integrated into the course materials using established instructional design principles.

In conclusion, we feel that it is important to design e-courses in such a manner as to force students to speak up and compel them to communicate with each other. Indeed, conversational teaching models, like those used in MBA courses, (Jung \& Suzuki, 2006) provide the much needed 'intellectual fodder' to fuel debate and interaction among students and teachers.

Still, we feel that because all undergraduate students must first learn the fundamentals of their discipline - fundamentals that tend to be imparted via more traditional 'teacher centric' models the use of 'conversational models' may not be fully appropriate for undergraduate e-courses delivered synchronously online. Indeed, unlike graduate students, undergraduate students typically have less time to devote to 'questioning' activities required to encourage responses 
(Koontz et al., 2006), simply because they are still absorbing the fundamentals. So, while sound design principle must clearly be used in all online synchronous courses, 'conversational models' may not be appropriate in introductory courses. Still, these very principles should be particularly helpful to help Japanese students overcome their cultural reluctance to draw attention to themselves by asking questions.

\section{Future Improvements}

Over time, we realized that students' hands-on use of Microsoft Excel helps them comprehend and master the online learning materials. Moreover, exercises in Microsoft Excel help to break up the e-class into manageable bits, breaking monotony of lectures and reducing eyestrain. Extra time spent for these online exercises during e-class hours is especially important for Japanese students, because they often do not have time to do review exercises unlike in the U.S. schools.

We also came to realize that using Microsoft PowerPoint for presentations often resulted in our teaching the class far too fast for our students to comprehend, probably because they remained passive instead of engaged learners. We also found that the online Introduction to Finance classes should be divided into different activities to include time for instruction, student exercises and presentations, and discussion. It is also important to determine the pacing and timing of classroom activities and ways to encourage students to participate. Finally, we found that free-flow discussions between and amongst students at the two locations made learning Introductory Finance a more fruitful learning experience.

Through two years of our experience in teaching the online Introductory Finance for undergraduate students, we have accumulated good assessments of the potential and the problems inherent in real time distance learning for the undergraduate students in Japan. It was a challenging task, but it was worthwhile trying. The mastery of the beginning level of Mathematics and Statistics is strongly called for learning of the Introductory Finance generally, and we expect that the use of the Microsoft Excel exercises in an exciting atmosphere of the real time distance e-Learning stands on its own foot at the frontier of the undergraduate education.

\section{Limitations and Conclusion}

The authors of this report are specialized in Economics and Finance. None had a formal academic background in Education or Pedagogy. Thus, a complete and thorough assessment of the effectiveness of this online teaching project was beyond our capacity. However, we did strive to analyze our 'practice' as based on the literature on instructional design principles. We have also highlighted unique characteristics facing e-Learning activities in Japanese higher education today. We therefore feel that the descriptive evidence reported here may benefit those seeking to do further research along this line of inquiry.

In conclusion, our experiment revealed several benefits and pitfalls of teaching synchronously at a distance, using e-Learning technologies. More importantly, we discovered and overcame numerous technological obstacles. As such, we found that technology itself was not basis of our most pressing concerns. Instead, we discovered that incorporating hands-on exercises (i.e., Microsoft Excel) into the online lectures provided an effective diversion for the student to remain engaged. We also found that efforts to maintain effective interaction between the two classrooms and asking students to prepare themselves by reading the course materials beforehand were the 
two factors that led to their success in this online course offered at the undergraduate level in Japan.

\section{Acknowledgement}

We thank Yusho Kagraoka for sharing the teaching experience, Makiko Miwa for helpful discussion, and Yoichi Konno for systems assistance.

\section{References}

Black, F., \& Scholes M. (1973). The pricing of options and corporate liabilities. Journal of Political Economy, 81, 637-659.

Chuo University (n.d.). Chuo University website. Retrieved March 12, 2007 from: http://www2.chuo-u.ac.jp/global/

ICU (n.d.). International Christian University website. Retrieved March 7, 2007 from: http://www.icu.ac.jp/index e.html

IT Strategic Headquarters (2001). IT Policy Package - 2005. Tokyo: Prime Minister of Japan and His Cabinet.

Jung, I., \& Suzuki, K. (2006). Blended learning in Japan and its application in liberal arts education. In C. J. Bonk \& C. R. Graham (Eds.) The Handbook of Blended Learning: Global perspectives, local designs. (pp.267-280). San Francisco: John Wiley and Sons \& Pfeiffer Publishing.

Kawachi, P. (2005a). Empirical validation of a multimedia construct for learning. In S. Mishra \& R. C. Sharma (Eds.) Interactive multimedia in education and training (pp. 158-183). Hershey, PA.: Idea Group.

Kawachi, P. (2005b). Open and Distance Education in Japan: implications for the rest of the world. In W. Y. Zhang (Ed.) Global Perspectives: Philosophy and practice in distance education, Vol. 3. Shanghai: China Central Radio and TV University Press.

Keio University (n.d.). Keio University website. Retrieved March 12, 2007 from: http://www.keio.ac.jp/

Koontz, F., Li, H., \& Compora, D. P. (2006). Designing Effective Online Instructions. Lanham, MD.: Rowman and Littlefield.

Kubota, K. (2001). Finance: Yoku Wakaru Finance [In Japanese]. Tokyo: Toyo-Keizai.

Shimizu, Y. (2005). Practical points of views for effective distance education and e-learning [in Japanese]. NIME Research Report, 1.: Chiba, Japan.

The Economist Intelligence Unit \& IBM Corporation (2003). 2003 e-Readiness Rankings. A White paper from The Economist Intelligence Unit. Retrieved November 26, 2006 from: http://graphics.eiu.com/files/ad_pdfs/eReady_2003.pdf 
Wong, S., \& Yoshida, A. (2001). Japan. In O. Jegede \& G. Shive (Eds.) Open and Distance Education in the Asia Pacific Region (pp. 80-102). Hong Kong: Open University of Hong Kong Press.

Yoshida, A. (2004). IT is in progress while e-learning is behind (Susumu IT ka to susumanu elearning) [In Japanese]. Newsletter of the National Institute of Multimedia Education. Chiba, Japan. 